\title{
Comparison of Cognitive-Affective Symptoms of Depression Between Cases of Low and High Suicidal Ideation in Medical Students
}

Nuzhat Aisha Akram, Nazia Jameel, Bushra Iftikhar, Tahira Assad, Farhan Muhammad Qureshi

ABSTRACT

Objective: To compare the severity of cognitive, affective and somatic symptoms of depression between two samples of medical students with high and low suicidal ideation using Beck Depression Inventory

Study Design and Setting: This is a cross sectional study conducted in public and private medical colleges in Karachi from September-December 2018.

Methodology: A total of 150 normal healthy students with 75 each from public and private medical colleges in Karachi were sampled through purposive sampling technique. Beck depression inventory (BDI) with twenty one items for depressive symptoms was used. Suicidal ideation was assessed using item 9 of BDI and participants were identified as having high and low suicidal ideation. Remaining twenty items of BDI were categorized as affective, cognitive and somatic symptoms of depression. Severity of each symptom was assessed through BDI score on a 4-point scale. Hypothesis testing for difference in BDI scores of depressive symptoms between high and low suicidal ideation cases was performed through independent sample t tests.

Result: BDI showed significantly higher suicidal ideation in public medical colleges' students. Cases with high suicidal ideation showed higher prevalence of five cognitive (past failure, feeling guilty, self-image, feeling of being punished, crying spells), four affective (sadness, irritability, decision making, self-dislike) and one somatic (physical health) symptoms of depression.

Conclusion: Significant higher prevalence of affective and cognitive symptoms of depression was found among cases of higher suicidal ideation.

Keywords: BDI, Cognitive affective symptoms, Medical students, Suicidal ideation.

\section{INTRODUCTION:}

Cases of suicidal ideation and suicidal behavior are growing among youth particularly among medical students ${ }^{1}$. Prevalence rate reported in literature for suicidal ideation is as high as $53.6 \%$ indicating the gravity of the situation ${ }^{2}$. Many factors have been associated with this grave illness, however, history of familial or non-familial psychiatric illness, loneliness, lack of social support, financial problems, problematic childhood, lack of parental care, abusive relationships and post-traumatic stress disorder (PSTD) are

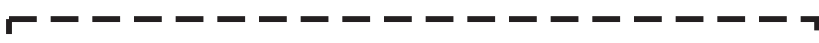

I Nuzhat Aisha Akram

I Assistant Professor, Department of Forensic Medicine and Toxicology, Karachi Institute of Medical Sciences, Karachi.

I Email: smr.akram@gmail.com.

I Nazia Jameel

Assistant Professor, Department of Community Medicine, I Baqai Medical University, Karachi.

I Bushra Iftikhar

I Assistant Professor, Department of Gynecology and Obstetrics, I Karachi Institute of Medical Sciences, Karachi.

Tahira Assad

Associate Professor, Department of Pharmacology,

I Karachi Institute of Medical Sciences, Karachi.

Farhan Muhammad Qureshi

I Assistant Professor, Department of Community Medicine,

I Karachi Institute of Medical Sciences, Karachi.

I Received: 09-07-2019

I Accepted: 15-11-2019

I - - - - - - - - - - - - - - - suggested as strong indicators of suicidal ideation which may end up in suicidal attempts or other suicidal behavior ${ }^{3,4,5,6}$.

Suicidal ideation is thought to be the preliminary step towards suicidal behavior ${ }^{7}$. Suicidal thoughts are considered as a persistent illness rather than an acute crisis. Therefore identification of associated risk factors for suicidal ideation and understanding the link between suicidal ideation and suicidal behavior is very important in order to design a preventive strategy against this growing illness. ${ }^{8,9}$ For this purpose research studies investigating suicidal ideation played a key role to understand this complex mental health issue.

Beck Depression Inventory, a self report measure is frequently used for the assessment of depression and depressive symptoms both in psychiatric and non-psychiatric healthy population ${ }^{10}$. BDI contains twenty one items which measure affective, cognitive and somatic symptoms of depression ${ }^{11}$. Affective symptoms of depression included in BDI are sadness, dissatisfaction and boredom, self-dislike, irritability, loss of interpersonal interest, indecisiveness. While cognitive symptoms included in BDI are pessimism, past failure, feeling guilty, feeling of being punished, self-blame, suicidal ideation, crying spells, self-image. Somatic symptoms included loss of working capability, sleep disturbance, physical energy, loss of appetite, However the validity of BDI as depicted by Cronbach's alpha is high for psychiatric patients than non-psychiatric healthy population ${ }^{12}$. Somatic symptoms of depression has been linked to diseases like 
heart failure, myocardial infarction and some metabolic syndromes ${ }^{13,14,15,16}$. Higher prevalence of somatic symptoms were also found in elderly population ${ }^{17,18}$ and female gender ${ }^{19}$. Some studies also revealed association of affective and cognitive symptoms with physical morbidity, however, somatic symptoms override cognitive and affective symptoms in such cases. ${ }^{20,21}$ Somatic symptoms like insomnia, lack of physical energy, insomnia, fatigue, weight loss have been linked to depression and suicidal ideation. ${ }^{9}$ Cases with high suicidal ideation have also showed impairments in cognitive control $^{22}$, that is they are unable to control their feelings and thoughts in order to understand the changes in environment and to cope with these changes. This impairment becomes pronounced when the environment is complex, challenging or ambiguous ${ }^{23}$.

Most of the depressive symptoms associated with suicidal ideation and suicidal behavior are affective and cognitive in nature ${ }^{11}$. Somatic factors like insomnia, fatigue etc are also commonly expressed through affective or cognitive symptoms. It was hypothesized that there is no significant difference in the prevalence of affective, cognitive, and somatic symptoms of depression between cases of high and low suicidal ideation. The current stud aimed to compare the prevalence of affective, cognitive and somatic symptoms using Beck Depression Inventory among cases of low and high suicidal ideation.

\section{METHODOLOGY:}

It was a cross sectional study conducted among third year medical students of public and private medical colleges in Karachi. The study participants were selected through purposive sampling technique. This study was approved by Ethical Review Board of Virtual University and Karachi Institute of Medical Sciences. Sample size was calculated using methodology given in Naing et $\mathrm{al}^{24}$ and at prevalence rate of $21 \% \%^{25}$ as 254 . Due to resource constraints 150 students were part of the study. Medical students of third year MBBS from both the genders and age between 18-24 years and healthy individuals were included in the study. All the cases with personal or familial history of psychiatric illnesses were excluded. Before giving the BDI the informed and written consent was obtained from the participants. Beck Depression Inventory was used to estimate the level of suicidal ideation and other depressive symptoms among medical students. Suicidal ideation among medical students was assessed using item 9 of BDI on 4-point scale ranging from $0-3$, where 0 means no suicidal thoughts and 3 means most severe suicidal thoughts. All other symptoms were also assessed on the same 4-point scale. Depressive symptoms were distributed into somatic, cognitive and affective categories as given in various studies. ${ }^{11,21}$ SPSS version 16 was used for data analyses and p-value $<0.05$ was considered as statistically significant. Independent t-tests was used to find out significant differences between the cases of high and low suicidal ideation across all the affective, cognitive and somatic depressive symptoms included in BDI. On the basis of BDI scores students from Public and Private medical colleges were now identified as cases of high and low suicidal ideation respectively.

\section{RESULTS:}

Suicidal Ideation was found in 58\% students of public medical colleges and only $11 \%$ of private medical colleges. Therefore samples from public medical colleges were identified as high suicidal ideation cases and those from private medical colleges as low suicidal ideation cases. BDI scores were calculated for each of the twenty items each of which depicted the severity of depressive symptoms on 4point scale ranging from 0 to 3 . BDI scores of high and low suicidal ideation cases differ across twenty items of Beck Depression Inventory- (Fig-1). This difference in BDI score was as high as 46 for working capability and 44 for crying spells to as low as 3 for loss of appetite.

Table 1 shows the items of BDI that were significant differences in BDI score between cases of high and low suicidal ideation.

\section{DISCUSSION:}

Depression is one of the major public health issues particularly of young students. Its prevalence rate is growing over the last two decades. Suicidal ideation is one of the major threatening symptoms of depression. According to World Health Organization suicide is the second leading cause of death among 15-29 years old. Variation reported in prevalence rate of depression and suicidal ideation in different studies can be attributed to differences in sample sizes, sampling area, study design, instrument or questionnaire used. Suicidal ideation is the most threatening symptom of depression that may lead to suicidal attempts and suicidal behavior. In our study the suicidal ideation was found in $58 \%$ and $11 \%$ students of public and private medical colleges respectively. On the other hand prevalence of sucidal ideation for medical students in Pakistan are as high as $31.4 \%$. The difference in rates of suicidal ideation may be atributed to factors mentioned above $\mathrm{e}^{26}$. Owing to its fatal consequences suicidal ideation must be investigated extensively so that prevention and intervention can be made promptly and properly. Although worldwide incidence rate of suicide is 800,000 , Pakistan has a lower rate of suicide which may result from under reported incidents, lack of research, or its status of being an offence in Pakistan Penal Code. ${ }^{27}$

The age shift of depression and suicidal ideation from elderly people to young generation was reported recently. Academic stress, family problems, financial stress, and competitive environment are held responsible for this illness ${ }^{6}$. In the context of Pakistan, a particular aspect of suicidal ideation and behavior is related to religion. Suicidal behavior is strictly prohibited in Islam. In spite of this religious prohibition prevalence is growing day by day showing the gravity of the problem and need for intervention through 
Fig- 1: BDI scores of depressive symptoms across high and low suicidal ideation cases of the present study.

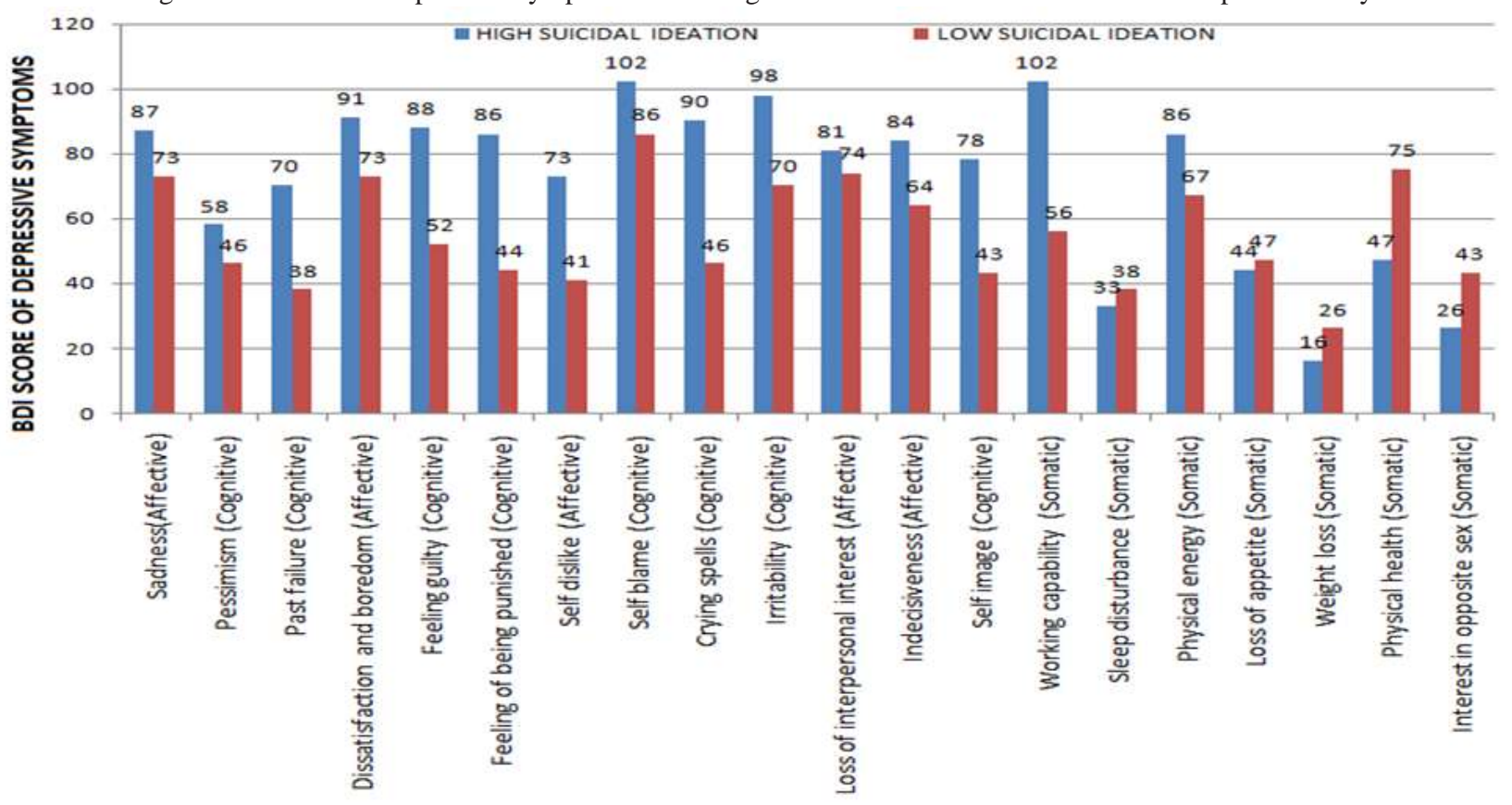

DEPRESSIVE SYMPTOMS OF BECK DEPRESSION INVENTORY (BDI)

Table-1: Independent sample $t$ tests showed significant differences ( $\mathrm{p}$ value $<0.05)$ in the prevalence of affective, cognitive and somatic symptoms between cases of high and low suicidal ideation.

\begin{tabular}{|c|l|c|c|c|}
\hline $\begin{array}{c}\text { BDI Item } \\
\text { No. }\end{array}$ & $\begin{array}{c}\text { Depressive symptoms included in Beck } \\
\text { Depression Inventory (BDI) }\end{array}$ & t value & $\begin{array}{c}\text { Degree of } \\
\text { Freedom }\end{array}$ & P-Value \\
\hline 1 & Sadness & 2.20 & 148 & 0.03 \\
\hline 3 & Past failure & 2.74 & 148 & 0.01 \\
\hline 5 & Feeling guilty & 3.87 & 148 & 0.00 \\
\hline 6 & Feeling of being punished & 2.86 & 148 & 0.01 \\
\hline 7 & Self-dislike & 2.88 & 148 & 0.01 \\
\hline 10 & Crying spells & 3.98 & 148 & 0.00 \\
\hline 11 & Irritability & 2.38 & 148 & 0.02 \\
\hline 13 & Indecisiveness & 2.12 & 148 & 0.04 \\
\hline 14 & Self-image & 2.15 & 148 & 0.03 \\
\hline 15 & Working capability & 4.69 & 148 & 0.00 \\
\hline 20 & Physical health & -2.68 & 148 & 0.01 \\
\hline
\end{tabular}

proper planning, research and collaborative efforts from all stake holders. For example, studies with a sample sizes ranging from 195 to 114 participants showed suicidal ideation as high as $38.7 \%$ (in Saudi Arabia) to as low as $17.5 \%$ (in Egypt $)^{25}$. It shows that religious beliefs make a little difference in suicidal ideation and it should be dealt with a broader perspective taking all the relevant factors into account. Prevention and intervention to this morbid illness need proper diagnosis. Health care professional must know that a patient showing depressive symptoms is vulnerable to develop suicidal ideation. Alarming symptoms and signs should be observed cautiously and must be investigated extensively through self-report measures, case history and in depth interviews. Some of the depressive cases report severe somatic symptoms and some are victims of cognitive and affective deterioration. However, studies revealed that patients who develop depression after a physical morbidity or illness are more likely to show somatic symptoms. These patients belong to elderly age group when physical illnesses are more common occurrence. Adolescents and young adults are more likely to develop affective and cognitive symptoms that usually precede the occurrence of full blown depression ${ }^{28}$. It is worth mentioning that cognitive symptoms are becoming the focus of interest for psychiatric illnesses other than 
depression like schizophrenia, bipolar disorders, etc. ${ }^{29,30}$ However; there is a dire need for exhaustive research to declare cognitive symptoms and dysfunction as causative factors or even risk factors for full blown depression, nevertheless association was revealed in various studies ${ }^{31}$. Cognitive and somatic symptoms have also been used for predicting biological changes in depression, reflecting the importance of assessing and diagnosing these symptoms in cases vulnerable to develop depression ${ }^{32}$. This study emphasized the importance of depressive symptoms and their distribution into somatic, cognitive and affective categories as the clinical picture of depression may vary with predominance of one or two of the three categories of depressive symptoms. Early intervention and preventive measures are likely to be more focused if the target groups can be identified and stratified according to the symptoms they predominantly present. As the suicidal ideation, the most threatening symptom of depression, is linked with suicidal behavior early diagnosis may help to reduce the suicide rates.

\section{CONCLUSION:}

Medical students were found more vulnerable to develop depressive symptoms. Significant higher prevalence of affective and cognitive symptoms of depression was found among cases of higher suicidal ideation.

\section{REFERENCES:}

1. Torres AR, Campos LM, Lima MCP, and Ramos-Cerqueira ATA. Suicidal ideation among medical students: prevalence and predictors. J Nerv Ment Dis. 2018; 206(3):160-168. doi: 10.1097/NMD.0000000000000734.

2. Coentre R and Góis C. Suicidal ideation in medical students: recent insights. Advances in Medical Education and Practice. 2018; 9: 873-880.

3. Carr ER, Woods AM, Vahabzadeh A, Sutton C, Wittenauer J andKaslow NJ. PTSD, depressive symptoms, and suicidal ideation in African American women: a mediated model. J Clin Psychol Med Settings. 2013; 20(1): . doi:10.1007/s10880012-9316-1.

4. Sobowale K, Zhou N, Fan J, Liu N, and Sherer R. Depression and suicidal ideation in medical students in China: a call for wellness curricula. Int J Med Educ. 2014;5:31-36.

5. Lamela D, Jongenelen I, Morais A and Figueiredo B. Cognitive-affective depression and somatic symptoms clusters are differentially associated with maternal parenting and coparenting. J Affect Disord. 2017;219:37-48. doi: 10.1016/j.jad.2017.05.006.

6. Cheema TN, Qasim AP, Munir U and Cheema FN. Changing trends in suicidal deaths over the past decade - an autopsy based study at Bahawalpur. APMC. 2018;12(2):154-7.

7. Syed EU, Asad N, Khan MM, Zaman M and Pirani S. On the pathway to suicide: suicidal ideation in young people in Karachi, Pakistan. Youth Voice J. 2018; 8.

8. Norr, AM, Allan NP, Macatee RJ, Capron DW and Schmidt NB. The role of anxiety sensitivity cognitive concerns in suicidal ideation: A test of the depression-distress amplification model in clinical outpatients. Psychiatry Research. 2016; 238: 74-80.
9. Allan NP, Conner KR, Pigeon WR, Gros DF, Salami TK and Stecker T. Insomnia and suicidal ideation and behaviors in former and current U.S. service members: Does depression mediate the relations? Psychiatry Research. 2017;252:296302. doi: 10.1016/j.psychres.2017.03.009.

10. Beck AT, Steer RA and Garbin M. Psychometric properties of the Beck Depression Inventory: twenty-five years of evaluation. Clin Psychol Review. 1988; 8:77-100.

11. Cheng HT, Ho M-C and Hung KY. Affective and cognitive rather than somatic symptoms of depression predict 3-year mortality in patients on chronic hemodialysis. Sci Rep. 2018; 8: 5868.doi: 10.1038/s41598-018-24267-5.

12. Beck AT and Steer RA. Manual for the Beck depression inventory 1993 edition. San Antonio (TX): The Psychological Corporation, 1987.

13. Angermann CE, Gelbrich G, Störk S, Schowalter M, Deckert J, Ertl G and Faller H. Somatic correlates of co-morbid major depression in patients with systolic heart failure. Int J Cardiol 2011;147(1):66-73. doi: 10.1016/j.ijcard.2009.07.044.

14. Groenewold NA, Doornbos B, Zuidersma M, Vogelzangs N, Penninx BWJH and Aleman Al. Comparing cognitive and somatic symptoms of depression in myocardial infarction patients and depressed patients in primary and mental health care. PLoS ONE. 2013;8(1): e53859. https://doi.org/10.1371/ journal.pone.0053859.

15. Benvenuti SM, Buodo G, Mennella R and Palomba D. Somatic, but not cognitive-affective, symptoms are associated with reduced heart rate variability in individuals with dysphoria. Front Psychol. 2015; doi: https://doi.org/10.3389/fpsyg.2015. 00599.

16. Wiltink J, Michal M, Jünger C, Münzel T, Wild PS, Lackner KJ, Blettner M, Pfeiffer N, Brähler E and Beutel ME. Associations between degree and sub-dimensions of depression and metabolic syndrome (MetS) in the community: results from the Gutenberg Health Study (GHS). BMC Psychiatry. 2018; 18:114. doi: https://doi.org/10.1186/s12888-018-16911.

17. Lesman-Leegte I, Jaarsma T, Coyne JC, Hillege HL, Van Veldhuisen DJ and Sanderman R. Quality of life and depressive symptoms in the elderly: a comparison between patients with heart failure and age- and gender-matched community controls. J Card Fail. 2009;15(1):17-23. doi: 10.1016/j.cardfail.2008.09. 006

18. Rutledge T, Reis VA, Linke SE, Greenberg BH and Mills PJ. Depression in heart failure; a meta-analytic review of prevalence, intervention effects, and associations with clinical outcomes.J Am Coll Cardiol. 2006; 48(8):1527-37.

19. Dessotte CAM, Silva FS, Furuya RK, Ciol MA, Hoffman JM and Dantas RAS. Somatic and cognitive-affective depressive symptoms among patients with heart disease: differences by sex and age. Rev. Latino-Am. Enfermagem. 2015;23(2):20815 doi: 10.1590/0104-1169.0287.2544.

20. Kupper, N, Widdershoven JW and Pedersen SS. Cognitive/ affective and somatic/affective symptom dimensions of depression are associated with current and future inflammation. J Affect Disord. 2011; doi:10.1016/j.jad.2011.10. 029.

21. Thombs BD, Ziegelstein RC, Pilote L, Dozois DJA, Beck AT, Dobson KS, Fuss S, Jonge P, Grace SL, Stewart DE, Ormel J and Abbey SE. Somatic symptoms overlap in Beck Depression Inventory-II scores following myocardial infarction. Br J Psychiatry. 2010; 197(1): 61-66. 
Nuzhat Aisha Akram, Nazia Jameel, Bushra Iftikhar, Tahira Assad, Farhan Muhammad Qureshi

22. Paulus MP. Cognitive control in depression and anxiety: out of control? Current Opinion in Behavioral Sci. 2015; 1:11320.

23. Huang H, Movellan J, Paulus MP, Harle KM. The influence of depression on cognitive control: Disambiguating approach and avoidance tendencies. PLoS One. 2015; 10 e0143714.

24. Naing L, Winn T, Rusli BN. Practical issues in calculating the sample size for prevalence studies. Archives of Orofacial Sci. 2006; 1: 9-14

25. Eskin M, Albuhairan F, Rezaeian M, Abdelkhalek AM, Harlak H, El-Nayal M, Asad N, et al. Suicidal thoughts, attempts and motives among university students in 12 muslim majority countries. Psych Qrtly. 2018; https://doi.org/10.1007/s11126018-9613-4.

26. Khokhar S and Khan MM. Suicidal ideation in Pakistani college students. Crisis 2005; 26(3):125-27.

27. Shekhani SS, Perveen S, Hashmi DS, Akbar K, Bachani S and Khan MM. Suicide and deliberate self-harm in Pakistan: a scoping review. BMC Psychiatry. 2018; 18:44. doi: 10.1186/s12888-017-1586-6.
28. Richardson L, Adams S and Metrics PX. Cognitive deficits in patients with depression. $\mathrm{J}$ for nurse practitioners 2018; 14(6):437-43. doi: https://doi.org/10.1016/j.nurpra.2018. 03.006 .

29. Ragguett, RM, Cha DS, Kakar R, Rosenblat JD, Lee Y and McIntyre RS. Assessing and measuring cognitive function in major depressive disorder. Evid Based Ment Health. 2016; 19: 106-9.

30. Zuckerman H, Pan Z, Park C, Brietzke E, Musial N, Shariq AS, Iacobucci M, Yim SJ, Lui LMW, Rong C and McIntyre RS. Recognition and treatment of cognitive dysfunction in major depressive disorder. Front Psychiatry. 2018; doi.org/10. 3389/fpsyt.2018.00655.

31. Scult MA, Paulli AR, Mazure ES, Moffitt TE, Hariri AR and Strauman TJ. The association between cognitive function and subsequent depression: a systematic review and meta-analysis. Psychol Med. 2016;14:1-17.

32. Dannehl K,Rief W, Schwarz MJ, Hennings A, Riemer $\mathrm{S}$,Selberdinger V, Stapf T and Euteneuer F. The predictive value of somatic and cognitive depressive symptoms for cytokine changes in patients with major depression. Neuropsychiatry Dis Treat. 2014; 10: 1191-1197. 